ISSN 1028-821X. Radiofiz. Electron. 2019. Vol. 24, No. 4: 63-69

DOI: https://doi.org/10.15407/rej2019.04.063

удК 621.396:621.391.82

PACS 42.30.sy

\title{
О. В. Сытник
}

Институт радиофизики и электроники им. А.Я. Усикова НАН Украины

12, ул. Акад. Проскуры, Харьков, 61085, Украина

E-mail: ssvp11@ire.kharkov.ua

\section{Особенности квазиоптимапьной дискретной обработки сигнапов}

Предмет и цель работы. Предметом исследования является система иифровой обработки сигналов радара, состоящчая из входного фильтра, аналого-ичифрового преобразователя и процессора, реализующего алгоритм обнаружения информационного сигнала в соответствии с некоторым наперед заданным критерием. Целью работы является оиенка потерь в отношении сигнал/шум при переходе от оптимальной к квазиоптимальной обработке сигналов с учетом спектральных характеристик сигналов и помех.

Методы и методология работы основаны на анализе спектральных функиий полезных сигналов и помех и вычислении ошибок их аппроксимации с помощью решетчатых функций при реальных длительностях выборок. Показано, что ошибки аппроксимаџии спектра полезного сигнала и спектра шума на выходе сглаживающего фильтра оказывают различное влияние на результат обработки сигнала. Для накопления сигнала и снижения уровня помех в реальном масштабе времени предложено путем выбора частоты, длительности отсчетов решетчатой функиии и ширины частотной характеристики фильтра минимизировать потери в отношении сигнал/иум при квазиоптимальной обработке сигналов.

Результаты работы. Получены аналитические соотношения для оиенок потерь в соотношении сигнал/иум при квазиоптимальной обработке сигналов по отношению к оптимальной. Показано, что при предельных частотах дополнительное снижение потерь можно получить путем изменения длительности выборки.

Заключение. Дискретная квазиоптимальная обработка сигналов при фиксированных длительностях выборок и полосах частот согласующих фильтров может приводить к значительным потерям в отношении сигнал/шум. Для снижения потерь необходимо находить компромисс между требованиями по быстродействию системы обработки, точности аппроксимации спектральных характеристик сигналов и помех и шириной полосы пропускания согласующего фильтра. Ил. 2. Библиогр.: 15 назв.

Ключевые слова: выборка, дискретная обработка, спектральная функция, частота дискретизации, решетчатая функция, алгоритм, критерий, бельй шум.

В подавляющем большинстве задач обработки сигналов различных радиоэлектронных систем остро стоит вопрос скорости получения конечной информации и простоты технических решений. Для получения решений в реальном масштабе времени зачастую приходится редуцировать оптимальные схемы обработки до квазиоптимальных. При этом возникает вопрос о потерях информации и о качестве принимаемых решений в сравнении с классическими оптимальными схемами.

Так, при построении реальных дискретных устройств обработки сигналов обычно приходится встречаться с условиями, когда ширина полосы спектральной плотности аддитивного шума превышает полосу частот, занимаемую полезным сигналом. Типичным примером такой ситуации является оптимальная обработка сигналов на фоне белого шума [1-3], когда невозможно не учитывать, что вообще дискретная обработка имеет смысл лишь при ограничении полосы частот, занимаемых непрерывным входным процессом. Такие ограничения возникают при объединении аналоговой и цифровой частей системы и осуществляются с помощью согласующего аналогового фильтра. В свою очередь, этот фильтр оказывает влияние не только на помеховую компоненту на- 
блюдаемого процесса, но и на спектр полезного сигнала. Это приводит к необходимости рассматривать задачу оптимальной дискретной обработки уже такого преобразованного сигнала на фоне преобразованного шума. Такая задача существенно отличается от классической задачи оптимального приема известного сигнала на фоне окрашенного шума $[4,5]$, решение которой можно свести к согласованной фильтрации сигнала на фоне декоррелирующего («обеляющего») аналогового фильтра, преобразующего окрашенный шум в белый.

Конечно, процедура декорреляции может быть выполнена в цифровой части системы с помощью специально сконструированного дискретного декоррелирующего фильтра, преобразующего случайную решетчатую функцию на выходе аналогового фильтра в случайную решетчатую функцию с равномерной спектральной плотностью. Такой фильтр усложняет обработку и повышает стоимость системы в целом. Поэтому интересно узнать, насколько велики энергетические потери при замене оптимального фильтра на квазиоптимальный дискретный фильтр.

1. Постановка задачи квазиоптимальной дискретно-аналоговой обработки сигналов. Пусть исследуется сигнал $s(t)$ на фоне гауссовского шума $n(t)$ с произвольной спектральной плотностью $G(\omega)$. Оптимальный дискретноаналоговый фильтр [6-8] должен содержать на входе аналоговый фильтр, ограничивающий полосу частот спектра входной реализации до полосы сигнала $s(t)$. На выходе этого фильтра спектральная плотность шума и сигнала будут $G_{1}(\omega)=G(\omega) H(\omega)$ и $S_{1}(\omega)=S(\omega) H(\omega)$ соответственно. Здесь $H(\omega)$ - передаточная функция аналогового фильтра.

Смесь полезного сигнала и шума дискретизируется в соответствии с теоремой Котельникова [9], в результате непрерывный процесс можно записать в виде решетчатой функции

$x_{1}[i]=s_{1}[i]+n_{1}[i], \quad i=1,2, \ldots$,

где $s_{1}[i]=s_{1}(i \Delta t), \quad n_{1}[i]=n_{1}(i \Delta t)$.

Корреляционная функция, описывающая шум, в общем случае, согласно [10], будет иметь вид

$$
R_{1}[l]=\frac{\Delta t}{2 \pi} \int_{-\pi / \Delta t}^{\pi / \Delta t} G_{1}(\omega) e^{j \omega l \Delta t} d \omega,
$$

где

$G_{1}(\omega)=\frac{1}{\Delta t} \sum_{k=-\infty}^{\infty} G\left(\omega+\frac{2 \pi}{\Delta t} k\right)\left|H\left(\omega+\frac{2 \pi}{\Delta t} k\right)\right|$.

Представим оптимальный по критерию максимума отношения сигнал/шум дискретный фильтр в виде последовательного соединения двух фильтров, первым из которых является дискретный декоррелирующий фильтр, преобразующий решетчатую функцию $x_{1}[i]$ в функцию $x_{2}[i]=x_{2}[i]+n_{2}[i]$ так, чтобы корреляционная функция $R_{2}[l]$ решетчатой функции $n_{2}[i]$ удовлетворяла условию

$R_{2}[l]= \begin{cases}1, & l=0 \\ 0, & l \neq 0 .\end{cases}$

Тогда задача построения оптимального дискретного фильтра сводится к задаче создания устройства, обеспечивающего обработку сигнала $s_{2}[i]$ на фоне шума, представленного некоррелированными выборочными значениями $n_{2}[i]$. В частности, если конечная цель обработки - обнаружение сигнала, то устройство обработки должно вычислять сумму [11]

$z_{2}=\sum_{i=1}^{N} x_{2}[i] s_{2}[i]$

и сравнивать ее с порогом, определяемым заданным критерием оптимальности.

2. Оценка отношения сигнал/шум. Отношение сигнал/шум $d_{0}^{2}$ на выходе устройства обработки определяется как отношение квадрата математического ожидания $E\left\{z_{2}\right\}^{2}$ и дисперсии $\operatorname{var}\left\{z_{2}\right\}$ величины $z_{2}$ при условии, что процесс $x_{2}[i]$ содержит полезный сигнал $s_{2}[i]$, т. е.

$d_{0}^{2}=E\left\{z_{2}\right\}^{2} / \operatorname{var}\left\{z_{2}\right\}$.

Используя соотношение Парсеваля для дискретных функций [11], можно записать выражение для квадрата математического ожидания $E\left\{z_{2}\right\}^{2}$ :

$E\left\{z_{2}\right\}^{2}=\sum_{i=1}^{N} s_{2}^{2}[i]=\frac{\Delta t}{2 \pi} \int_{-\pi / \Delta t}^{\pi / \Delta t}\left|F_{2}^{*}(j \omega)\right|^{2} d \omega$,

где $F_{2}(j \omega)$ - спектр решетчатой функции $s_{2}[i]$, определяемый как

$F_{2}(\omega)=\frac{1}{\Delta t} \sum_{k=-\infty}^{\infty} S\left(\omega+\frac{2 \pi}{\Delta t} k\right) H\left(\omega+\frac{2 \pi}{\Delta t} k\right)$. 
Из формулы (8) явный вид квадрата математического ожидания $E\left\{z_{2}\right\}^{2}$ можно представить как

$$
\begin{aligned}
& E\left\{z_{2}\right\}^{2}=\frac{1}{2 \pi} \times \\
& \times \int_{-\pi / \Delta t}^{\pi / \Delta t} \frac{\left|\sum_{k=-\infty}^{\infty} S\left(\omega+\frac{2 \pi}{\Delta t} k\right) H\left(\omega+\frac{2 \pi}{\Delta t} k\right)\right|^{2}}{\sum_{k=-\infty}^{\infty} G\left(\omega+\frac{2 \pi}{\Delta t} k\right)\left|H\left(\omega+\frac{2 \pi}{\Delta t} k\right)\right|} d \omega .(9)
\end{aligned}
$$

Числитель подынтегрального выражения в формуле (9) на интервале частот $|\omega| \leq \pi / \Delta t$ представляет собой эквивалентный спектр $S_{1 e q}(\omega)$ соответствующего непрерывного процесса $s_{1 e q}(t)$, представленного решетчатой функцией $s_{1}[i]$. Аналогично знаменатель подынтегрального выражения формулы (9) есть спектральная плотность $G_{1 e q}(\omega)$ эквивалентного непрерывного шумового процесса $n_{1 e q}(t)$, представленного решетчатой функцией $n_{1}[i]$.

Тогда

$$
E\left\{z_{2}\right\}^{2}=\frac{1}{2 \pi} \int_{-\pi / \Delta t}^{\pi / \Delta t} \frac{\left|S_{1 e q}[\omega]\right|^{2}}{G_{1 e q}(\omega)} d \omega,
$$

где

$$
\begin{aligned}
& S_{1 e q}(\omega)=\left\{\begin{array}{l}
\sum_{k=-\infty}^{\infty} S\left(\omega+\frac{2 \pi}{\Delta t} k\right) H\left(\omega+\frac{2 \pi}{\Delta t} k\right), \\
|\omega| \leq \frac{\pi}{\Delta t}, \\
0, \quad|\omega| \geq \frac{\pi}{\Delta t} ;
\end{array}\right. \\
& G_{1 e q}(\omega)=\left\{\begin{array}{l}
\sum_{k=-\infty}^{\infty} G\left(\omega+\frac{2 \pi}{\Delta t} k\right) H\left(\omega+\frac{2 \pi}{\Delta t} k\right), \\
|\omega| \leq \frac{\pi}{\Delta t}, \\
0, \quad|\omega| \geq \frac{\pi}{\Delta t} .
\end{array}\right.
\end{aligned}
$$

Очевидно, что в случае дельта-коррелированного шума дисперсия

$$
\operatorname{var}\left\{z_{2}\right\}=\frac{1}{N-1} \sum_{i=1}^{N} s_{2}^{2}[i] .
$$

Сравнивая (13) и (5), из (6) с учетом (10) получим соотношение сигнал/шум

$$
d_{0}^{2}=\frac{1}{2 \pi} \int_{-\pi / \Delta t}^{\pi / \Delta t} \frac{\left|S_{1 e q}(\omega)\right|^{2}}{G_{1 e q}(\omega)} d \omega .
$$

В реальных условиях ни спектр сигнала, ни частотная характеристика фильтра не являются финитными функциями частоты. Поэтому для квазиоптимальной системы обработки сигналов, в которой условия (11) и (12) выполняются лишь частично, следует сделать оценку проигрыша в отношении сигнал/шум, обусловленную наличием спектральных компонент, не удовлетворяющих этим условиям.

3. Анализ потерь в отношении сигнал/шум квазиоптимальной системы обработки сигналов. В реальных системах при адекватном выборе частоты дискретизации [12] отличие эквивалентного непрерывнозначного случайного процесса $n_{1 e q}(t)$ от соответствующего реального процесса $n_{1}(t)$ достаточно мало, поэтому спектр шума можно приближенно представить как

$$
\begin{aligned}
& G_{1 e q}(\omega) \approx G(\omega)|H(\omega)| \times \\
& \times\left(\begin{array}{l}
1-G^{-1}(\omega)|H(\omega)|^{-1} \times \\
\left.\times \sum_{\substack{k=-\infty \\
k \neq 0}}^{\infty} G\left(\omega+\frac{2 \pi}{\Delta t} k\right)\left|H\left(\omega+\frac{2 \pi}{\Delta t} k\right)\right|\right)^{-1} .
\end{array}\right.
\end{aligned}
$$

Числитель подынтегральной функции в (14) можно оценить следующим образом:

$$
\begin{aligned}
& \left|S_{1 e q}(\omega)\right|^{2} \geq|S(\omega) H(\omega)|^{2} \times \\
& \times\left(\begin{array}{l}
1-\frac{2}{|S(\omega) H(\omega)|} \times \\
\times \sum_{\substack{k=-\infty \\
k \neq 0}}^{\infty}\left|S\left(\omega+\frac{2 \pi}{\Delta t} k\right) H\left(\omega+\frac{2 \pi}{\Delta t} k\right)\right|
\end{array}\right)^{-1} .
\end{aligned}
$$

Учитывая (15) и (16), из (14) получаем

$d_{0}^{2} \geq d_{00}^{2}-d_{01}^{2}-d_{02}^{2}$,

где

$d_{00}^{2}=\frac{1}{2 \pi} \int_{-\pi / \Delta t}^{\pi / \Delta t} \frac{|S(\omega)|^{2}}{G(\omega)} d \omega$, 


$$
\begin{aligned}
& d_{01}^{2}=\frac{1}{\pi} \int_{-\pi / \Delta t}^{\pi / \Delta t} \frac{S(\omega)}{G(\omega)|H(\omega)|} \times \\
& \times \sum_{\substack{k=-\infty \\
k \neq 0}}^{\infty}\left|S\left(\omega+\frac{2 \pi}{\Delta t} k\right) H\left(\omega+\frac{2 \pi}{\Delta t} k\right)\right| d \omega, \\
& d_{02}^{2}=\frac{1}{2 \pi} \int_{-\pi / \Delta t}^{\pi / \Delta t} \frac{|S(\omega)|^{2}(\omega)\left|H^{2}(\omega)\right|}{G^{2}} \times \\
& \times \sum_{\substack{k=-\infty \\
k \neq 0}}^{\infty}\left|G\left(\omega+\frac{2 \pi}{\Delta t} k\right) H\left(\omega+\frac{2 \pi}{\Delta t} k\right)\right|^{2} d \omega .
\end{aligned}
$$

Отличие отношений сигнал/шум на выходе устройства оптимальной дискретной обработки и аналогового согласованного фильтра характеризуется энергетическим проигрышем

$\gamma_{0}=h_{0}^{2} / d_{0}^{2}$,

где $h_{0}^{2}=\frac{1}{N_{0} \pi} \int_{-\infty}^{\infty}|S(\omega)|^{2} d \omega$ для аналогового фильтра нижних частот с прямоугольной амплитудно-частотной характеристикой и полосой пропускания $|\pi / \Delta t| ; N_{0}-$ спектральная плотность белого шума.

Для того чтобы воспользоваться соотношением (18) для оценки энергетических потерь квазиоптимальной системы по отношению к оптимальной, необходимо конкретизировать ряд характеристик системы. В частности, определим величину потерь (18) для системы, содержащей аналоговый фильтр Баттерворта [13], частотная характеристика которого аппроксимируется функцией вида

$$
H(\omega)=\left\{\begin{array}{l}
1,|\omega| \leq 2 \pi F_{H}, \\
\left(2 \pi F_{H} /|\omega|\right)^{m},|\omega| \geq 2 \pi F_{H}, m>1,
\end{array}\right.
$$

где $F_{H}-$ верхняя частота пропускания фильтра.

Относительно спектра сигнала введем следующее ограничение:

$$
|S(\omega)| \leq \frac{B}{|\omega|^{n}}, \quad|\omega|>2 \pi F_{S},
$$

где $B$ - некоторая константа; $F_{S}-$ верхняя частота спектра сигнала.

Сигналы со спектральной функцией вида (20) характерны, например, для процессов дыхания и сердцебиения $[14,15]$.

66
Рассмотрим область значений $\Delta t$, определяемую условиями

$$
\begin{aligned}
& \pi / \Delta t>2 \pi F_{S}, \\
& \pi / \Delta t>2 \pi F_{H} .
\end{aligned}
$$

Следует отметить, что на практике порядок фильтра $m$ не превосходит $n$, т. е. $m \leq n$. Перепишем соотношение (18) с учетом (17)

$\gamma_{0} \leq \frac{1}{d_{00}^{2} / h_{0}^{2}-d_{01}^{2} / h_{0}^{2}-d_{02}^{2} / h_{0}^{2}}$.

Проанализируем компоненты, входящие в знаменатель выражения (22):

$d_{00}^{2} / h_{0}^{2} \geq 1-\left(2 F_{S} \Delta t\right)^{2 n-1} /(2 n-1)$

$d_{01}^{2} / h_{0}^{2} \leq \frac{2}{m+n-1}\left(2 F_{H} \Delta t\right)^{m+n-1} \times$

$\times\left(\left(F_{S} / F_{H}\right)^{n-1}+\left(F_{S} / F_{H}\right)^{2 n-1}\right)$;

$d_{02}^{2} / h_{0}^{2} \leq\left(2 F_{H} \Delta t\right)^{2 m-1} \times$

$\times\left(1+\left(F_{S} / F_{H}\right)^{2 n}\right)\left[(2 m-1)\left(F_{S} / F_{H}\right)\right]^{-1}$.

Подставляя (23)-(25) в (22), получаем

$\gamma_{0}^{-1} \geq 1-\frac{\left(2 F_{S} \Delta t\right)^{2 n-1}}{2 n-1}-$

$-\frac{2\left(2 F_{H} \Delta t\right)^{m+n-1}}{m+n-1}\left(1+\left(F_{S} / F_{H}\right)^{n}\left(F_{S} / F_{H}\right)^{n-1}-\right.$

$\left.-\frac{\left(2 F_{H} \Delta t\right)^{2 m-1}}{(2 m-1)\left(F_{S} / F_{H}\right)}\left(1+\left(F_{S} / F_{H}\right)^{2 n}\right)\right)$.

На рис. 1 и 2 показаны кривые потерь, рассчитанные в соответствии с (26) при различ-

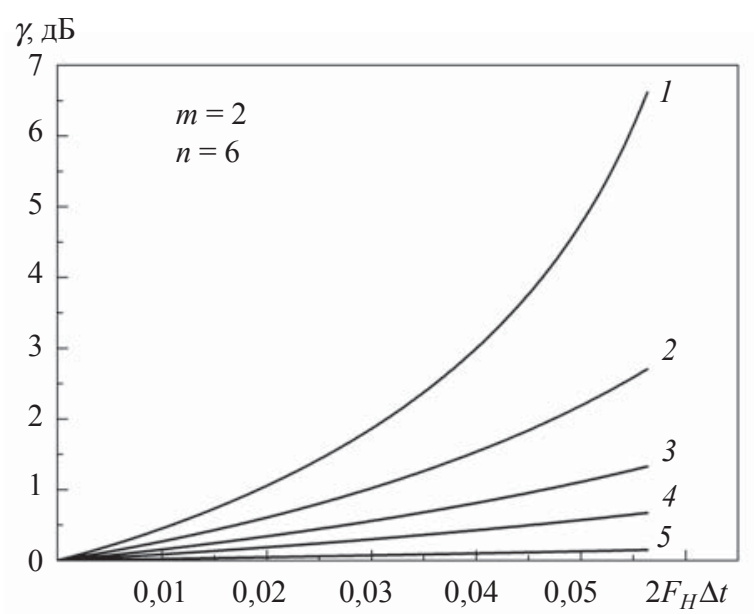

Puc. 1. Кривые энергетических потерь квазиоптимальной системы по отношению к оптимальной при $m>n$

ISSN 1028-821X. Radiofiz. Electron. 2019. Vol. 24, No. 4 


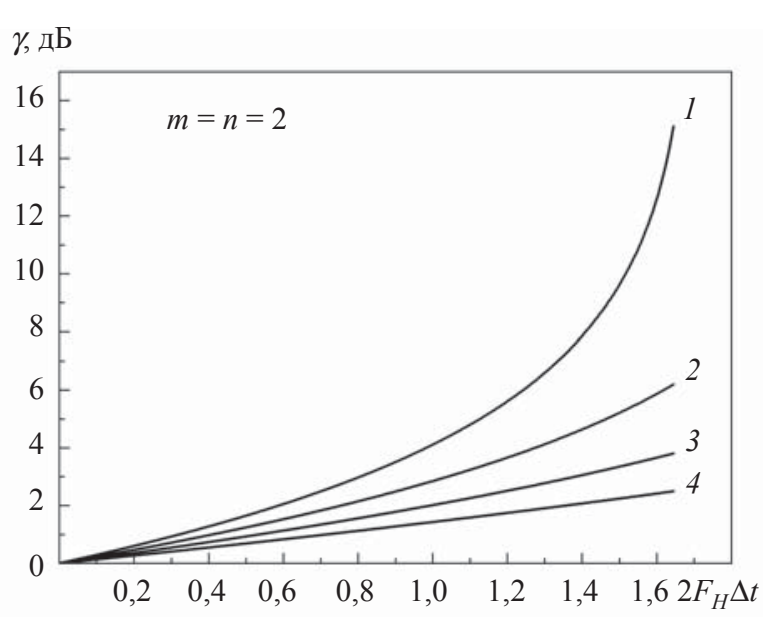

Puc. 2. Кривые энергетических потерь квазиоптимальной системы по отношению к оптимальной при $m=n$

ных соотношениях параметров сигналов и характеристиках системы обработки.

В частности, кривая 1 на рис. 1 получена при отношении $F_{S} / F_{H}$, равном 1,4 , кривая $2-$ при 1,3 , кривая 3 - при 1,2, кривая 4 - при 1,1 , кривая 5 при - 1,0 соответственно. Априорно полагалось, что порядок фильтра $m=2$, а скорость убывания частотных компонент спектра сигнала в (20) $n=6$. На рис. 2 показаны такие же зависимости, но при $m=n=2$ и отношении $F_{S} / F_{H}$, равном 1,1 для кривой $1,1,0$ для кривой 2, 0,9 для кривой 3 и 0,8 для кривой 4 соответственно.

Выводы. Как следует из зависимостей рис. 1 и 2, величина энергетических потерь сущест- венно зависит от параметров входного фильтра. Так, при $F_{S} \leq F_{H}$ и высоком порядке фильтpa $m \geq 6$ приближение длительности интервала дискретизации $\Delta t$ к величине $1 / 2 F_{H}$ приводит к резкому увеличению энергетического проигрыша квазиоптимальной системы по отношению к оптимальной. Для фильтров низкого порядка $m \leq 3$ интервал $\Delta t$, выбранный с запасом по отношению к величине $1 / 2 F_{H}$, т. е. $\Delta t<(0,6 \ldots 0,7) / 2 F_{H}$, обеспечивает вполне приемлемые потери при обработке сигналов квазиоптимальной системой.

Очевидно, что при фиксированных значениях величин $\Delta t$ и $F_{H}$ энергетические потери в отношении сигнал/шум возрастают с увеличением частоты $F_{S}$. При наличии технических возможностей эти потери можно скомпенсировать уменьшением интервала дискретизации. В конечном итоге при обработке информационных входных сигналов в дискретном виде необходимо, несмотря на наличие сглаживающего фильтра, ограничивающего полосу частот аналогового входного сигнала, учитывать так же и значение полосы частот сигнала до фильтра при выборе интервала дискретизации. Физическое объяснение этому можно найти в эффекте частичной декорреляции отсчетов, вызванной влиянием фильтра. При заранее известных величинах $F_{S}, F_{H}, m$ и $n$ можно путем выбора интервала $\Delta t$ минимизировать потери в отношении сигнал/шум.

\section{БИБЛИОГРАФИЧЕСКИЙ СПИСОК}

1. Левин Б.Р. Теоретические основы статистической радиотехники: в 3-х кн. Москва: Сов. радио, 1977. Кн. 1. 608 с.

2. Тихонов В.И. Статистическая радиотехника. Москва: Сов. радио, 1982. 624 с.

3. Крамер Г., Лидбеттер М. Стационарные случайные процессы. Свойства выборочных функций и их приложения: моногр. Пер. с англ. Ю.К. Беляева, М.П. Ершова. Москва: Мир, 1969. 400 с.

4. Ван Трис Г. Теория обнаружения оценок и модуляц̧ии: в 3-х кн. Пер. с англ. под ред. В.И. Тихонова. Москва: Сов. радио, 1972. Кн. 1. 744 с.

5. Dixon W.G., Massey F.J. Introduction to Statistical Analysis. N.Y.: Mc Graw-Hill, 1968. 536 p.

6. Otnes R.K., Enochson L. Applied Time Series Analysys. N.Y.: J. Willey, 1982. 428p.

7. Голд Б., Рэйдер Ч.М. Цифровая обработка сигналов. Пер. с англ. под ред. А.М. Трахтмана. Москва: Сов. радио, 1973.368 с.

8. Biguesh M., Gershman A. Training-based MIMO Channel Estimation: a Study of Estimator Tradeoffs and Optimal Training Signals. IEEE Trans. Signal Process. 2006. Vol. 54, Iss. 3. P. 884-893. DOI: 10.1109/TSP.2005.863008.

9. Lo N., Falconer D.D., Sheikh, A.H. Adaptive Equalization and Diversity Combining for Mobile Radio Using Interpolated Channel Estimates. IEEE Trans. Veh. Technol. 2018. Vol. 40, Iss. 3. P. 636-645. DOI: 10.1109/25.97518.

10. Гоноровский И.С. Радиотехнические иели и сигнальл. Москва: Радио и связь, 1986. 512 с.

11. Proakis J., Salehi M. Digital Communications. $5^{\text {th }}$ ed. McGraw-Hill Higher Education, 2013.118 p.

12. Lathi B.P., Ding Z. Modern Digital and Analog Communication Systems. $4^{\text {th }}$ ed. Oxford University Press, 2019.325 p.

13. Sytnik O.V., Vyazmitinov I.A., Myroshnychenko Ye.I., Kopylov Yu.A. Spectral Selection of Very-Low Frequencies Processes. Telecommunications and Radio Engineering. 2009. Vol. 68, N 2. P. 137-144. DOI: 10.1615/TelecomRadEng.v68.i2.50.

14. Сытник О.В., Вязьмитинов И.А., Мирошниченко Е.И. Статистические свойства спектральных оценок информационных сигналов при зондировании малоподвижных объектов. Физические основы приборостроения. 2012. Т. 1, № 4(5). C. 78-85. DOI: 10.25210/jfop-1204-078085. 
15. Sytnik O.V. Invariant Transformation in Identification Theory. Telecommunications and Radio Engineering. 2003. Vol. 60, N 10-12. P. 20-32. DOI: 10.1615/TelecomRadEng.v60.i1012.30.

\section{REFERENCES}

1. Levin, B.R., 1977. Theoretical Foundations of Statistical Radio Engineering. Moscow: Sov. Radio Publ. B. 1. (in Russian).

2. Tihonov, V.I., 1982. Statistical Radio Engineering. Moscow: Sov. Radio Publ. (in Russian).

3. Kramer, G., Lidbetter, M., 1969. Stationary and related stochastic processes. Sample function properties and their applications. Translated from English by Yu.K. Belyaev, M.P. Ershov. Moscow: Mir Publ. (in Russian).

4. Van Trees, H.L., 1972. Detection, Estimation, and Modulation Theory. Translated from English and ed. by V.I. Tikhonov. Moscow: Sov. Radio Publ. Pt. 1. (in Russian).

5. Dixon, W.G., Massey, F.J., 1968. Introduction to Statistical Analysis. Mc Graw-Hill, N.Y.

6. Otnes, R.K., Enochson, L., 1982. Applied Time Series Analysys. J. Willey, N.Y.

7. Gold, B., Rader, C.M., 1973. Digital Processing of Signals. Translated from English and ed. by A.M. Trakhtman. Moscow: Sov. Radio Publ. (in Russian).

8. Biguesh, M., Gershman, A., 2006.Training-based MIMO Channel Estimation: a Study of Estimator Tradeoffs and Optimal Training Signals. IEEE Trans. Signal Process., 54(3), pp. 884-893. DOI: 10.1109/TSP.2005.863008.

9. Lo, N., Falconer, D.D., Sheikh, A.H., 2018. Adaptive Equalization and Diversity Combining for Mobile Radio Using Interpolated Channel Estimates. IEEE Trans. Veh. Technol., 40(3), pp. 636-645. DOI: 10.1109/25.97518.

10. Gonorovsky I. S. Radio engineering circuits and signals. Moscow: Sov. Radio, 1978. 304 p. (in Russian).

11. Proakis, J., Salehi, M., 2013. Digital Communications. $5^{\text {th }}$ ed. McGraw-Hill Higher Education.

12. Lathi, B.P., Ding, Z., 2019. Modern Digital and Analog Communication Systems. $4^{\text {th }}$ ed. Oxford University Press.

13. Sytnik, O.V., Vyazmitinov, I.A., Myroshnychenko, Ye.I., Kopylov, Yu.A., 2009. Spectral Selection of Very-Low Frequencies Processes. Telecommunications and Radio Engineering, 68(2), pp. 137-144. DOI: 10.1615/TelecomRadEng.v68.i2.50.

14. Vyazmitinov, I.A., Myroshnichnko, Ye.I., Sytnik, O.V., 2012. Statistical Properties of Spectral Estimates of Information Signals for Sounding of Slowly Moving Targets. Physical Bases of Instrumentation, 1(4(5)), pp. $78-85$ (in Russian). DOI: 10.25210/jfop-1204-078085.

15. Sytnik, O.V., 2003. Invariant Transformation in Identification Theory. Telecommunications and Radio Engineering, 60(1012), pp. 20-32. DOI: 10.1615/TelecomRadEng.v60.i1012.30.

Received 18.03.2019

O.V. Sytnik

O.Ya. Usikov IRE of NASU

12, Acad. Proskura St., Kharkiv, 61085, Ukraine

\section{FEATURES OF QUASI-OPTIMAL DISCRETE SIGNAL PROCESSING}

Subject and Purpose. The research subject is a digital processing system of radar signals. The system consists of an input filter, an analog-to-digital converter and a processor that implements an algorithm of information signal detection in accordance with some previously given criterion. The aim of the work is the loss estimation in the signal-to-noise ratio on a change-over from the optimal signal processing to the quasi-optimal technique, the spectral characteristics of signals and the interference being considered.

Methods and Methodology. Analysis is performed for the spectral functions of useful signals and noise figures combined with the error calculation results concerning these functions approximations using lattice functions with real sample durations. It is shown that the approximation errors of the useful signal spectrum and the noise spectrum at the smoothing filter output exert different effects on the signal processing result. In order to build up the signal and reduce the noise level in real time, it is proposed to minimize losses in the signal-to-noise ratio as applied to the quasi-optimal signal processing by fitting the frequency, the duration of lattice function samples and the width of the filter frequency response.

Results. Analytical relations have been obtained for loss estimation in the signal-to-noise ratio in the case of quasi-optimal signal processing compared to the optimal one. It is shown that at the cutoff frequencies, some additional loss reduction can be obtained by varying the sampling duration.

Conclusion. The discrete quasi-optimal signal processing at fixed sample durations and in certain frequency bands of the matching filters can give significant signal-to-noise losses. To reduce these losses, a compromise has to be reached between the operation speed of the processing system, the approximation accuracy of the spectral characteristics of signals and noise figures, and the matching filter passband.

Key words: sampling, discrete processing, spectral function, sampling frequency, lattice function, algorithm, criterion, white noise. 


\section{O.B. Ситнік}

IPE ім. О.Я. Усикова НАН України

12, вул. Акад. Проскури, Харків, 61085, Україна

\section{ОСОБЛИВОСТІ КВАЗІОПТИМАЛЬНОГО ДИСКРЕТНОГО ОБРОБЛЕННЯ СИГНАЛІВ}

Предмет і мета роботи. Предметом дослідження є система цифрового оброблення сигналів радара, яка складається 3 вхідного фільтра, аналого-цифрового перетворювача і процесора, що реалізує алгоритм виявлення інформаційного сигналу відповідно до деякого наперед заданого критерію. Метою роботи є оцінка втрат у відношенні сигнал/шум при переході від оптимального до квазіоптимального оброблення сигналів з урахуванням їх спектральних характеристик.

Методи і методологія роботи засновані на аналізі спектральних функцій корисних сигналів і завад, а також обчисленні помилок їх апроксимації за допомогою гратчастих функцій при реальних тривалостях виборок. Показано, що помилки апроксимації спектра корисного сигналу і спектра шуму на виході згладжувального фільтра мають різний вплив на результат обробки сигналу. Для накопичення сигналу і зниження рівня завад у реальному масштабі часу запропоновано шляхом вибору частоти, тривалості відліків гратчастої функції і ширини частотної характеристики фільтра мінімізувати втрати у відношенні сигнал/шум при квазіоптимальному обробленні сигналів.

Результати роботи. Отримано аналітичні співвідношення для оцінок втрат у співвідношенні сигнал/шум при квазіоптимальному обробленні сигналів відносно оптимального. Показано, що при граничних частотах додаткове зниження втрат можна отримати шляхом зміни тривалості вибірки.

Висновки. Дискретне квазіоптимальне оброблення сигналів при фіксованих тривалостях виборок і смугах частот узгоджувальних фільтрів може призводити до значних втрат у відношенні сигнал/шум. Для зменшення втрат необхідно знаходити компроміс між вимогами щодо швидкодії системи оброблення, точності апроксимації спектральних характеристик сигналів і завад та шириною смуги пропускання узгоджувального фільтра.

Ключові слова: вибірка, дискретне оброблення сигналів, спектральна функиія, частота дискретизаиії, тратчаста функція, алгоритм, критерій, білий шум. 